\title{
Linearization of Anti-Tank Guided Missile
}

\section{Mohamed Abdallah Mohamed, Ahmed Nasr Ouda, Bahaaeldin Gamal Abdelaty, Ahmed Hassan*}

Electrical Engineering Department, Technical Research Center, Cairo, Egypt

\section{Email address:}

mam0001@yahoo.com (M. A. Mohamed), ahnasroda@yahoo.com (A. N. Ouda), bahaa.blackeagel@yahoo.com (B. G. Abdelaty), ahmed_hassan_aircraft@yahoo.com (A. Hassan)

${ }^{*}$ Corresponding author

\section{To cite this article:}

Mohamed Abdallah Mohamed, Ahmed Nasr Ouda, Bahaaeldin Gamal Abdelaty, Ahmed Hassan. Linearization of Anti-Tank Guided Missile. Automation, Control and Intelligent Systems. Vol. 7, No. 1, 2019, pp. 9-17. doi: 10.11648/j.acis.20190701.12

Received: February 25, 2019; Accepted: April 11, 2019; Published: May 17, 2019

\begin{abstract}
Anti-tank surface to surface guided missile systems are considered to be one of the most powerful weapons in the modern war. Due to their effectiveness against either fortifications or tanks in addition to their relative low weight, they are deployed in the field easily. One of the most challenges the engineers face while designing a guided missile is the autopilot design. The autopilot loop or the inner loop of the missile should steer the missile during its spatial flight till hitting the target even it is moving or stationary. A higher maneuver target was hit by the missile, the more reliable autopilot you design. On designing a mature autopilot, a promising mathematical model of the missile should be in hand and mathematical linearized model should be derived. This paper presents a mathematical representation of an anti-tank guided missile the mathematical linearized model as well so that the design of the missile autopilot is presented in the future work.
\end{abstract}

Keywords: Guided Missiles, Autopilot, Linearization, Anti-tank, Classical Controller, Transfer Function

\section{Introduction}

The intended missile system uses the command line of sight (CLOS) guidance method to hit its target even this target is moving or stationary [10]. The operator has to keep the sight reticle on the target center and as a result, missile spatial position is measured and sensed via the sight or night detectors located in the ground station, the miss angle between the actual missile position and the virtual line of sight is calculated in missile guidance unit (MGU) and consequently it generates the steering commands sent to the missile via the command wire [1]. These commands are received by the on-board missile electronics and in turn the missile control surface deflects according the angel commanded resulting in a new missile position. The actual angle carried out by the missile in yaw plane is measured by the free gyro and the difference between the achieved missile angle and the commanded angle sent via the wire $(\Delta \psi)$ is compensated by the inner loop compensation network as shown in figure 1 [1].

The inner loop or the autopilot loop is designed to compensate the error between command wire angle and the achieved missile angle as shown in figure 2 [1].

The motivation of linearizing the missile model is that it is a must to design a mature controller capable of dealing with maneuvering targets. The controller should be mature enough to deal with all flight conditions [14]. In the first section the coordinate systems and the total forces and moment acting on the missile are presented as well. The 6-DOF model of the missile is derived in the second section while the third section introduce the linearization methodology. The paper ends with conclusion and references in the fourth and fifth section respectively. 


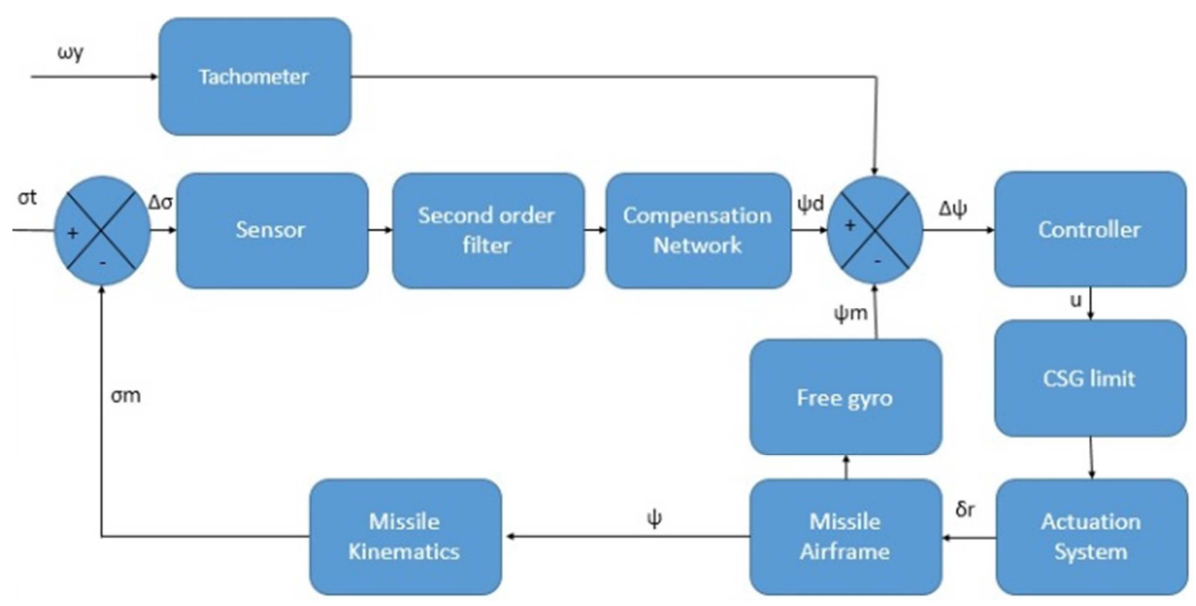

Figure 1. Bloch diagram of yaw channel.

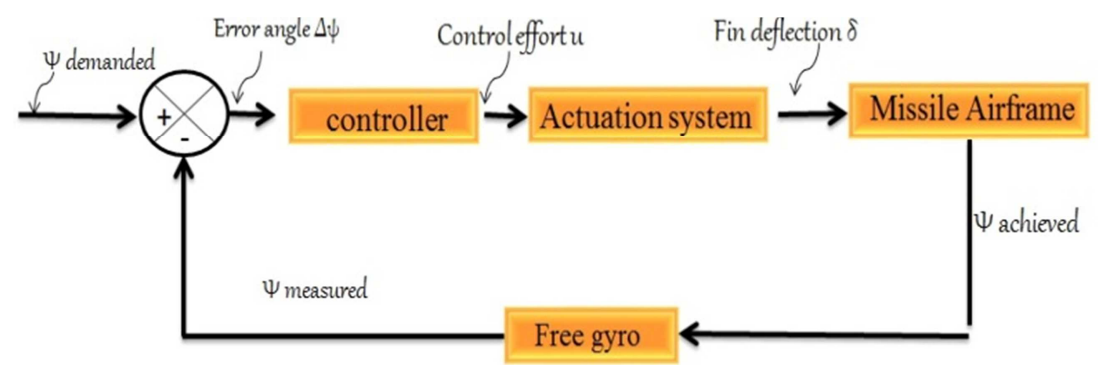

Figure 2. The autopilot loop.

\section{Missile Coordinate Systems}

The vehicle motion is described in terms of a time varying position vector and its time derivatives that require a reference frame with respect to which position, velocity and acceleration are measured [2]. The choice of the reference frame depends on the system design taking into consideration the computation frame work and the measurements of necessary parameters. There are three main types of coordinate systems [3]:

i Fixed (ground) coordinate system (G.C.S). ( $\left.X_{g} Y_{g} Z_{g}\right)$

ii Board (movable or body) coordinate system (B.C.S). $\left(\mathrm{O} \mathrm{X}_{1} \mathrm{Y}_{1} \mathrm{Z}_{1}\right)$

iii Velocity coordinate system (V.C.S). (OXYZ)

\subsection{Fixed (Ground) Coordinate System (G.C.S)}

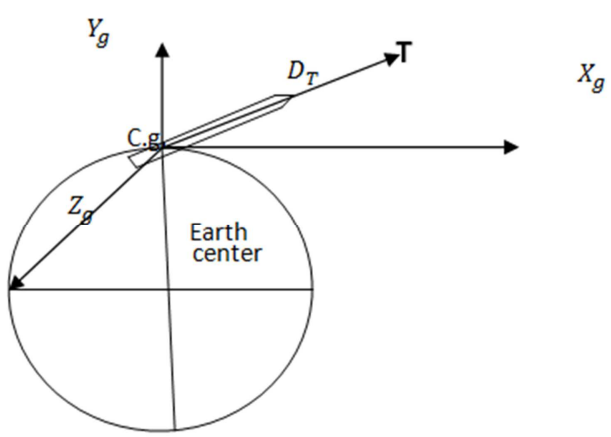

Figure 2. Ground coordinate system.

The Ground coordinate system shown in Figure 2. is used to relate the missile motion with respect to the Earth's surface where the origin of (G.C.S) is position at the missile center of gravity at the instant of lunching, the $\mathrm{Xg}$-axis is parallel to the reference passing through the launch site and is position toward the launch site, the $\mathrm{Yg}$-axis is normal to reference and is positive upward and the $\mathrm{Zg}$-axis standard right-handed orthogonal system [4].

\subsection{Board (Moveable or Body) Coordinate System}

It is local coordinate system it is used to describe the motion of missile relative of the ground coordinate system. This system is attached missile airframe as shown in figure 3 . The origin is missile center of gravity (c.g), the $X_{1}$ is aligned with missile longitudinal, $Y_{1}$ is aligned to be perpendicular to axis $X_{1}$ and lies in the plane of missile and axis $Z_{1}$ is oriented to be system clockwise rectangular one [standard righthanded system, 5].

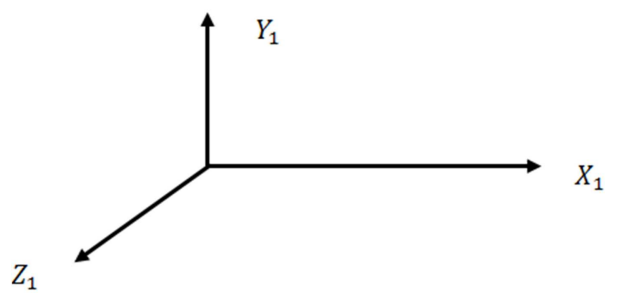

Figure 3. Body coordinate system.

\subsection{Velocity Coordinate System (V.C.S)}

Velocity coordinate system shown in figure 4 is used to 
characterize the direction of missile flight and to simplify the aerodynamic calculations. The origin of this system is in the missile center of gravity (c.g), X-axis conjugate with the direction of missile velocity vector $\left[{ }^{-} \mathrm{V}_{\mathrm{m}}\right], \mathrm{Y}$-axis has direction of the main normal to the path and $\mathrm{Z}$-axis completes a standard right-handed system [6].

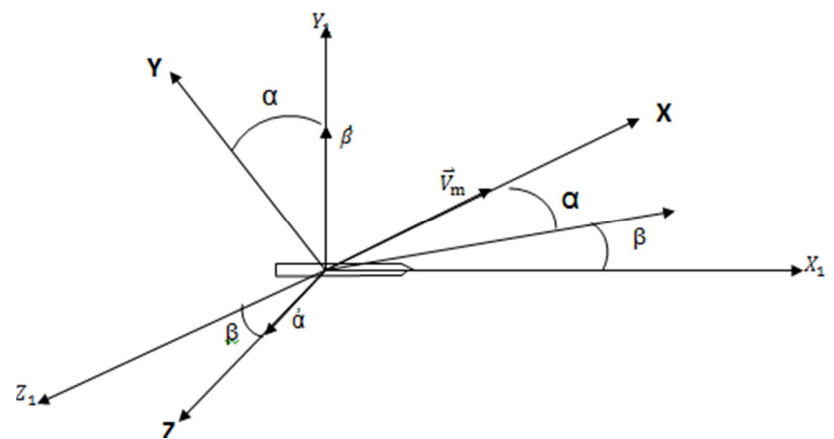

Figure 4. Velocity coordinate system.

The mutual position between different coordinate system can be represented by using the direction cosines approach is used where the transformation matrix $(\mathrm{T})$ relates two right hand orthogonal coordinate system. In this approach, the matrix of coordinate transformation is composed of the cosines of angles included by the axes of the two coordinated system [6].

\subsection{Board-Ground Coordinate System}

The body and ground reference frames are related to each other by Euler's angles $(\psi, \vartheta, \gamma)$ as shown in figure 5 .

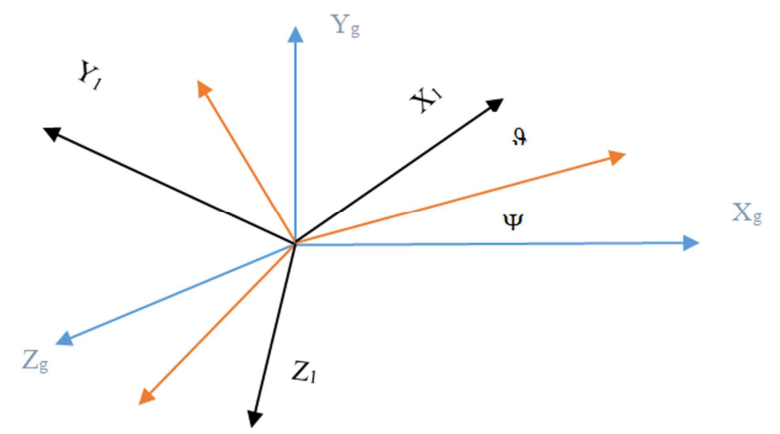

Figure 5. Board-Ground Coordinate System.

The transformation of coordinates from the body coordinate system into the ground coordinate system can be carried out using the following matrix [7]:

$$
\left[\begin{array}{l}
x_{g} \\
y_{g} \\
z_{g}
\end{array}\right]=\left(\begin{array}{ccc}
\cos \psi \cos \vartheta & \sin \psi \sin \gamma-\cos \psi \sin \vartheta \cos \gamma & \sin \psi \cos \gamma+\cos \psi \sin \vartheta \sin \gamma \\
\sin \vartheta & \cos \gamma \cos \vartheta & -\cos \vartheta \sin \gamma \\
-\cos \vartheta \sin \psi & \cos \psi \sin \gamma+\sin \psi \sin \vartheta \cos \gamma & \cos \psi \cos \gamma-\sin \psi \sin \vartheta \sin \gamma
\end{array}\right)\left[\begin{array}{c}
x_{1} \\
y_{1} \\
z_{1}
\end{array}\right]
$$

\subsection{Velocity-Ground Coordinate System}

The velocity and ground reference frames are related to each other by Euler's angles $(\varphi, \theta, \chi)$ as shown in figure 6 .The transformation of coordinates from the body coordinate system into the ground coordinate system can be carried out using the following matrix [8]

$$
\left[\begin{array}{l}
x_{g} \\
y_{g} \\
z_{g}
\end{array}\right]=\left(\begin{array}{ccc}
\cos \varphi \cos \theta & \sin \varphi \sin \chi-\cos \varphi \sin \theta \cos \chi & \sin \varphi \cos \chi+\cos \varphi \sin \theta \sin \chi \\
\sin \theta & \cos \theta \cos \chi & -\cos \theta \sin \chi \\
-\sin \varphi \cos \theta & \cos \varphi \sin \chi+\sin \varphi \sin \theta \cos \chi & \cos \varphi \cos \chi-\sin \varphi \sin \theta \sin \chi
\end{array}\right)\left[\begin{array}{l}
x \\
y \\
z
\end{array}\right]
$$

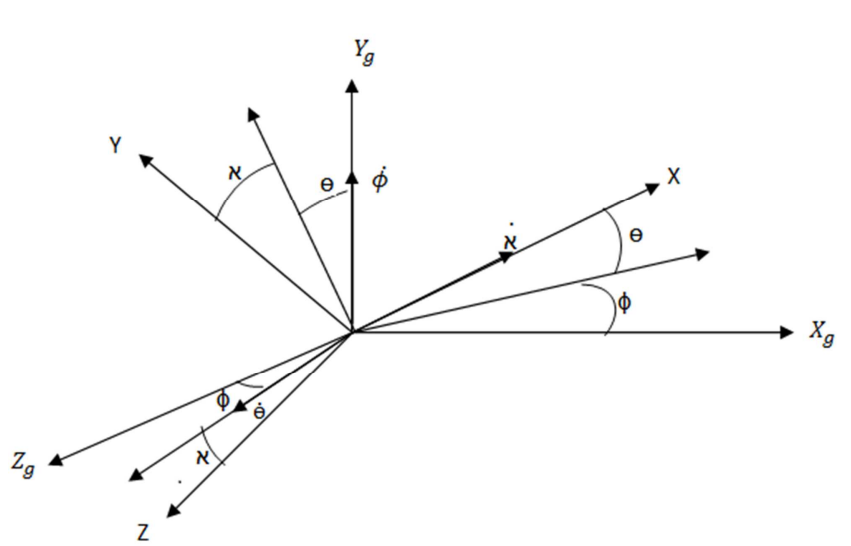

Figure 6. Velocity-Ground Coordinate system.

\subsection{Body-Velocity Coordinate System}

The body and velocity reference frames are related to each other by Euler's angles $(\alpha, \beta)$ as shown in figure 7 . The transformation of coordinates from the body coordinate system into the velocity coordinate system can be carried out using the following matrix [9]:

$$
\left[\begin{array}{l}
x \\
y \\
z
\end{array}\right]=\left(\begin{array}{ccc}
\cos \beta \cos \alpha & -\cos \beta \sin \alpha & \sin \beta \\
\sin \alpha & \cos \alpha & 0 \\
-\sin \beta \cos \alpha & \sin \beta \sin \alpha & \cos \beta
\end{array}\right)\left[\begin{array}{l}
x_{1} \\
y_{1} \\
z_{1}
\end{array}\right]
$$




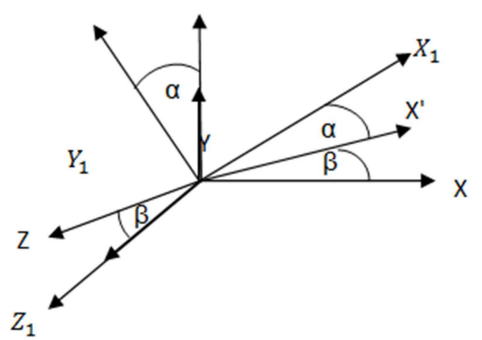

Figure 7. Body-Velocity Coordinate system.

\section{Six Degree of Freedom Missile Model}

The design and analysis of a real system necessitates the availability of a mathematical model, either in state-space or in transfer function format. This mathematical model is a set of differential equations that describe the missile motion [7]. The missile motion is represented by the forces and moments acting upon it during flight. These forces and moments necessitate availability of the aerodynamic force and moment coefficients, it is necessary to know all the time the coordinates of both target and the missile w.r.t the control point in order to be able to guide the missile towards target. In anti-tank command guidance system an operator at the guidance or control point solves the mission of interception on the basis of obtained coordinates and forms the command, according to the utilized or specified guidance method. Then these commands are sent to the control system on the missile for changing its spatial position. The guidance unit compares the two set of data (missile and target positions) and issues the appropriate correction (guidance command) according to the employed guidance method to the missile during its flight through the wire. The equations of missile motion are derived according to Newton's second law which states that the summation of all external forces acting on a body is equal to the time rate of change of its velocity. In addition, the summation of the external moments acting on the missile body is equal to the time rate of change of the momentum, which is mean angular momentum [7].

Acting forces and moment on missile fuselage

The total forces acting on the missile body can be categorized into three main forces; thrust force $(\mathrm{T})$, missile weight $(\mathrm{G})$ and the aerodynamic forces (drag force, lift force and side force) [1]. These forces can be expressed as follows [10]:

$$
\begin{gathered}
\mathrm{T}=\mathrm{T}_{\mathrm{x} 1} \\
\overline{G=} m_{s} \bar{g} \\
X=-C_{x} S q \\
Y=C_{y} S q \\
Z=-C_{z} S q
\end{gathered}
$$

The main affecting moment on the missile body ( $\mathrm{X}$ form missile type) is the aerodynamic moment. The aerodynamic moment originates from the aerodynamic forces that don't pass through the missile C.G. This moment has three components; pitch, yaw and roll moments. Usually the aerodynamic moment is given by its components along the axes of board coordinate system as follows [10]:

$$
\begin{aligned}
& M_{a_{x 1}}=m_{x 1} l_{x} S q \\
& M_{a_{y 1}}=m_{y 1} l_{y} S q \\
& M_{a_{z 1}}=m_{z 1} l_{z} S q
\end{aligned}
$$

The 6 DOF model of the underlying missile can be expressed as follows [10]:

$$
\begin{gathered}
\dot{V}_{x 1}=\frac{T_{x}}{m}-\frac{C_{x} S q}{m}-g \sin \vartheta+V_{y 1} \omega_{z 1}-V_{z 1} \omega_{y 1} \\
\dot{V}_{y 1}=\frac{C_{y} S q}{m}-g \cos \vartheta \cos \gamma+V_{z 1} \omega_{x 1}-V_{x 1} \omega_{z 1} \\
\dot{V}_{z 1}=-\frac{C_{z} S q}{m}-g \cos \vartheta \sin \gamma+V_{x 1} \omega_{y 1}-V_{y 1} \omega_{x 1} \\
\dot{\psi}=\left(\omega_{y 1} \cos \gamma-\omega_{z 1} \sin \gamma\right) \cos e c \vartheta \\
\dot{\vartheta}=\omega_{y 1} \sin \gamma+\omega_{z 1} \cos \gamma \\
\dot{\gamma}=\omega_{x 1}-\left(\omega_{y 1} \cos \gamma-\omega_{z 1} \sin \gamma\right) \tan \vartheta
\end{gathered}
$$

\section{Linearization of the Missile Model}

One of the most common problems faced by the designer of a classical controllers is that of translating the missile tactical problem into specifications for the control system design. These specifications necessitate the availability of the system transfer function in conjunction with the appropriate autopilot $[11,12]$.

Missile Air frame Transfer function

At the commencement of the study of the missile control system designs. One must make certain assumptions. A traditional assumption is one of the linearity the hardware and the equations of motion. The transfer function is a mathematical representation in S-domain between Euler's angles $(\theta, \psi, \varphi)$ and the command $(\delta)$ function in the aerodynamic coefficients. And finally, the missile may well have to operate over a range of speeds and angles of attack. A representative number of these operating conditions is also tested in function of frequency and the missile control system is designed counting on an intermediate operating point of these operating conditions and if the design is satisfactory at this point, then it is satisfactory at all the operating points [13].

Pitch plane transfer Function $(\vartheta / \delta \mathrm{p})$ :

Pitch plane transfer function is a relation between missile pitch angle $(\vartheta)$ and the fin command in pitch plane $(\delta p)$. From figure 6 the angular velocity components of the velocity coordinate system (VCS) are given as follows [10]:

$$
\begin{gathered}
\Omega_{x}=\dot{\chi}+\dot{\varphi}^{*} \sin \theta_{M} \\
\Omega_{y}=\dot{\varphi}_{M} * \cos \theta_{M} * \cos \chi+\dot{\theta}_{M} * \sin \chi
\end{gathered}
$$




$$
\Omega_{z}=\dot{\theta_{M}} * \cos \chi-\dot{\varphi_{M}} * \cos \theta_{M} * \sin \chi
$$

Multiplying equations (10) by $\sin (\chi)$ and equation (12) by $\cos (\chi)$ and adding both equation yielding

$$
\begin{gathered}
\dot{\theta}_{M}=\Omega_{y} * \sin \chi+\Omega_{z} * \cos \chi \\
\dot{\varphi_{M}}=\left(\Omega_{y} * \cos \chi-\Omega_{z} * \sin \chi\right) / \cos \theta_{M} \\
\dot{\varphi_{M}}=\Omega_{x}-\left(\Omega_{y} * \cos \chi-\Omega_{z} * \sin \chi\right) * \tan \theta_{M}
\end{gathered}
$$

But according to reference [10]

$$
\begin{aligned}
& F_{x}=m * \dot{V}_{M} \\
& F_{y}=m * V_{M} * \omega_{z 1}=m * V_{M} * \Omega_{z} \\
& F_{z}=-m * V_{M} * \omega_{y 1}=-m * V_{M} * \Omega_{y}
\end{aligned}
$$

Then

$$
\Omega_{y}=-F_{z} / m^{*} V_{M}
$$

And

$$
\Omega_{z}=F_{y} / m^{*} V_{M}
$$

Then from equations (17) and (18)

$$
\begin{aligned}
& \Omega_{y}=-g_{z} / V_{M}+\left(1 / m^{*} V_{M}\right) *\left(T^{*} \sin \beta^{*} \cos \alpha+C_{z} * S^{*} q\right) \\
& \Omega_{z}=g_{y} / V_{M}+\left(1 / m^{*} V_{M}\right) *\left(T^{*} \sin \alpha+C_{y} * S^{*} q\right)
\end{aligned}
$$

Substituting from equations (19) and (20) in equation (13)

$$
\begin{aligned}
& \dot{\theta_{M}}=-g / V_{M}-K^{*} \sin \chi+N^{*} \cos \chi \\
& \text { where: } \\
& K=\frac{-T^{*} \cos \alpha * \sin \beta}{m^{*} V_{M}}-\left(\frac{C_{z} * S^{*} q}{m^{*} V_{M}}\right), \\
& N=\frac{T^{*} \sin \alpha}{m^{*} V_{M}}+\left(\frac{C_{y} * S^{*} q}{m^{*} V_{M}}\right),
\end{aligned}
$$

For simplicity the following assumptions are take into consideration:

(1) Constant missile velocity.

(2) Neglecting gravity.

(3) Small angle of attack and side slip angel such that $\cos (\alpha)=\cos (\beta)=1$ and $\sin (\alpha)=\sin (\beta)=0$.

Applying these assumptions on the above equation yields

$$
\dot{\theta_{M}}=\left(\frac{T+C_{y}^{\alpha} * S^{*} q}{m^{*} V_{M}}\right) * \alpha+\left(\frac{C_{y}^{\delta_{p}} * S^{*} q}{m * V_{M}}\right) * \delta_{p}
$$

But

$$
\begin{aligned}
& \dot{\vartheta}=\omega_{y 1} \sin \gamma+\omega_{z 1} \cos \gamma \\
& \text { so } \\
& \dot{\vartheta}=\omega_{z 1}
\end{aligned}
$$

And

$$
\ddot{\vartheta}=\dot{\omega}_{z 1}=\frac{M_{z 1}}{I_{z z}}
$$

So

$$
\ddot{\vartheta}=\left(\frac{S^{*} q^{*} l}{I_{z z}}\right) *\left(m_{z 1}{ }^{\alpha} * \alpha+m_{z 1} \dot{\vartheta} * \dot{\vartheta}+m_{z 1}{ }_{p} * \delta_{p}\right)
$$

The geometrical relation:

$$
\alpha=\theta_{M}-\vartheta
$$

Substituting from equation (25) in (22) yields

$$
\alpha=\frac{n_{\delta_{p}} * \delta_{p}+s \vartheta}{s-n_{\alpha}}
$$

Where:

$$
\begin{aligned}
& n_{\alpha}=\left(\frac{T+C_{y}{ }^{\alpha} * S * q}{m * V_{M}}\right), \\
& n_{\delta_{p}}=\left(\frac{C_{y}{ }^{\delta_{p}} * S * q}{m * V_{M}}\right)
\end{aligned}
$$

Substituting from equation (26) in equation (24) yields the pitch plane transfer function:

$$
\frac{\vartheta}{\delta_{p}}=\frac{\left(-M_{z 1}^{\delta_{p}}\right) * s+\left(M_{z 1}^{\delta_{p}} * n_{\alpha}-M_{z 1}^{\alpha} * n_{\delta_{p}}\right)}{-s^{3}+\left(n_{\alpha}+M_{z 1}^{\dot{\vartheta}}\right) * s^{2}-\left(n_{\alpha} * M_{z 1}^{\dot{\vartheta}}+M_{z 1}^{\alpha}\right) * s}
$$

Where:

$$
\begin{aligned}
& M_{z 1}^{\delta_{p}}=\frac{m_{z 1}^{\delta_{p}} * S * q * l}{I_{z z}}, \\
& M_{z 1}^{\alpha}=\frac{m_{z 1}^{\alpha} * S * q * l}{I_{z z}}, \\
& M_{z 1}^{\dot{\vartheta}}=\frac{m_{z 1}^{\dot{\vartheta}} * S * q * l}{I_{z z}}
\end{aligned}
$$

Yaw Plane Transfer Function ( $\psi / \delta y)$

Similar to the pitch plane; yaw transfer function is a relation between missile yaw angle $(\psi)$ and the fin command in pitch plane $(\delta y)$.By applying the above procedures for yaw plane we can simply get the following equations [10]

$$
\dot{\varphi}=n_{\beta} * \beta+n_{\delta_{y}} * \delta_{y}
$$

Where:

$$
\begin{aligned}
& n_{\beta}=\left(\frac{T+C_{z}^{\beta *} S^{*} q}{m * V_{M}}\right), \\
& n_{\delta_{y}}=\left(\frac{C_{z}^{\delta_{y} *} S^{*} q}{m^{*} V_{M}}\right)
\end{aligned}
$$


And;

$$
\ddot{\psi}=M_{y 1}^{\beta} * \beta+M_{y 1}^{\delta_{y}} * \delta_{y}+M_{y 1}^{\dot{\psi}} * \dot{\psi}
$$

Finally, the yaw plane transfer function is

$$
\frac{\psi}{\delta_{y}}=\frac{\left(-M_{y 1}^{\delta_{y}}\right) * s+\left(M_{y 1}^{\delta_{y}} * n_{\beta}-M_{y 1}^{\beta} * n_{\delta_{y}}\right)}{-s^{3}+\left(n_{\beta}+M_{y 1}^{\dot{\psi}}\right) * s^{2}-\left(n_{\beta} * M_{y 1}^{\dot{\psi}}+M_{y 1}^{\beta}\right) * s}
$$

Where:

$$
\begin{aligned}
& M_{y 1}^{\delta_{y}}=\frac{m_{y 1}^{\delta_{y}} * S^{*} q * l}{I_{y y}}, \\
& M_{y 1}^{\beta}=\frac{m_{y 1}^{\beta} * S^{*} q * l}{I_{y y}}, \\
& M_{y 1}^{\dot{\psi}}=\frac{m_{y 1}^{\dot{\psi}} * S^{*} q * l}{I_{y y}}
\end{aligned}
$$

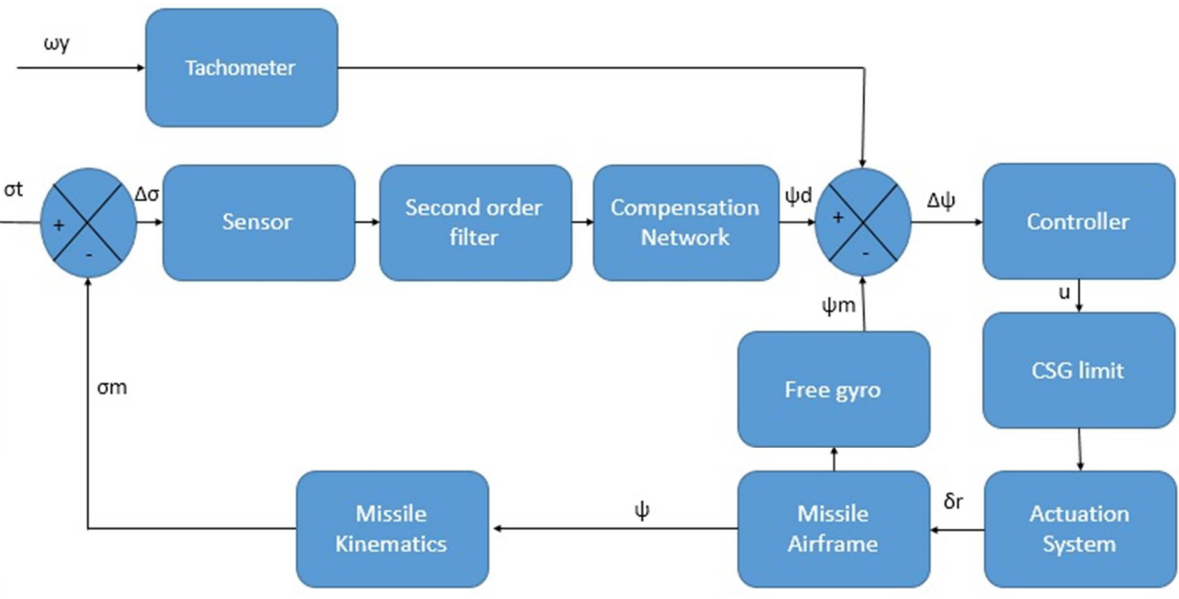

Figure 8. Block diagram of Yaw Plane.

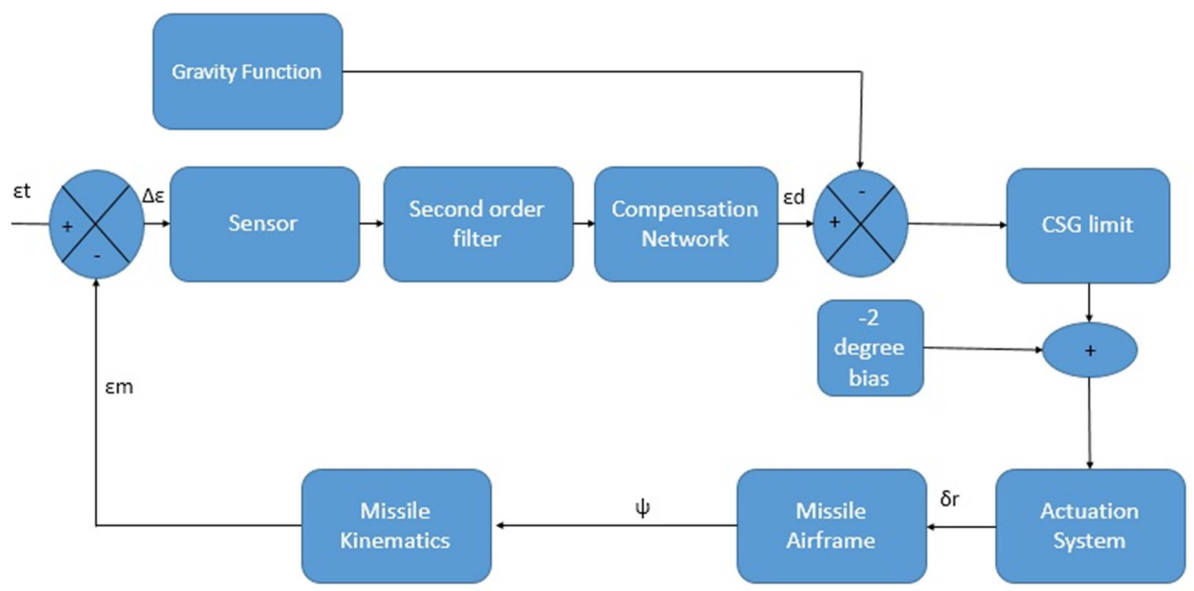

Figure 9. Block diagram of Pitch Plane.

\subsection{Compensation Network}

The compensation network is a series of filters whose purpose is to minimize the effect of system transients and system error sources such as thrust misalignments, control

\section{Missile Modelling}

The design and analysis of a real system necessitates the availability of a mathematical model, either in state-space or in transfer function format. This mathematical model is a set of differential equations that describe the missile motion. The block diagram of both yaw and pitch planes represented as set of transfer functions are shown in figure 8 and figure 9 [14].

\subsection{Sensor and Error Detectors}

The intended missile is controlled by measuring the angular distance between the missile xenon source or the thermal beacon and the line of sight. The sensor and error detectors measure the missile error angle in pitch from the electronic bore sight using the day sight and the night sight as shown in figure 8 and figure 9 . In some cases this angular error sensed by the guidance algorithm is weighted average of the two trackers and for the analysis of the two channels [14]. 
varying gravity bias function to produce the missile control surface command. In yaw channel, the filter output is summed with the azimuth tachometer input and a time varying open loop steering function [14].

\subsection{Command Signal Generator Limit (CSG)}

The summation of tachometer rate, g-bias, and yaw open loop steering and compensation network outputs is limited via the command signal generator limits. The purpose of this limit functions is twofold. First, the commanded missile angle of attack must not exceed the structural capabilities of the airframe, and second to ensure that the command signal must be below levels at which excessive coupling between the steering loops and the roll channel could occur [14].

\subsection{CVAC Compensation}

Due to gravity and changing missile velocity, the commanded control surface duty cycle in pitch doesn't operate around zero. However, due to bandwidth limitations in the command link, a sine wave carrier must be used. As the duty cycle varies from $50 \%$ the sine wave amplitude of the carrier changes the open loop gain. The control signal after being summed with a sine wave carrier in the launcher is sent to the missile via the command link. In the yaw channel, this composite signal is summed with the roll command and yaw rate command from the onboard missile attitude gyro before being detected by yaw zero-crossing detectors. In the pitch channel, this signal is summed with the roll attitude command before being detected. Each of the four rectangular signals from the zero-crossing detectors activates a corresponding control surface through a solenoid valve and pneumatic actuator in the missile. This operates the missile control surfaces; two in each plane. The required aerodynamic moments are obtained by controlling the relative duration of the time period (duty cycle) in which the control surfaces are at their travel limits [14].

\subsection{Control Surfaces}

The missile control surfaces are four rectangular plates located in the aft part of the missile deflecting horizontally and vertically yielding the controlling aerodynamic forces and moment acting on the missile airframe. The flippers on the missile are driven by a bang-bang actuator system. Thus for an ideal flipper model, the flipper will always be at an extreme position [14].

\section{Simulation Results}

The pitch and yaw block diagrams are programmed under MATLAB environment to investigate the behavior of the linear system against step response input represents the position of the target. Figure 10 and 11 show (a) the sensor output, (b) the compensating network output, (c) the second order filter output and finally in (d) the missile response due to unit step input.
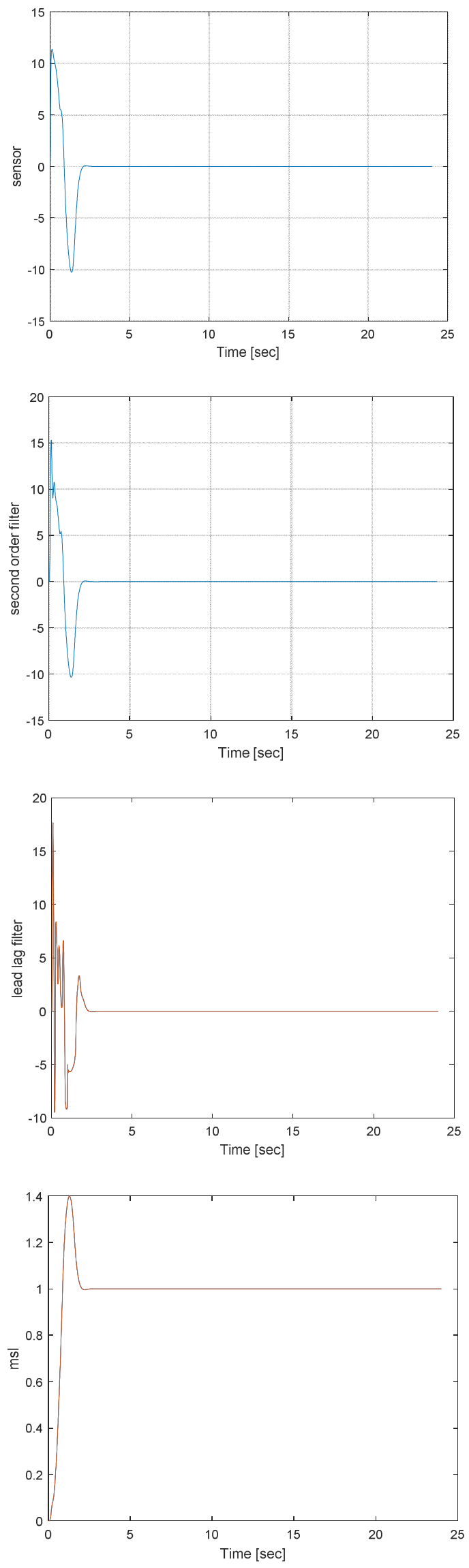

Figure 10. Pitch Plane output responses. 

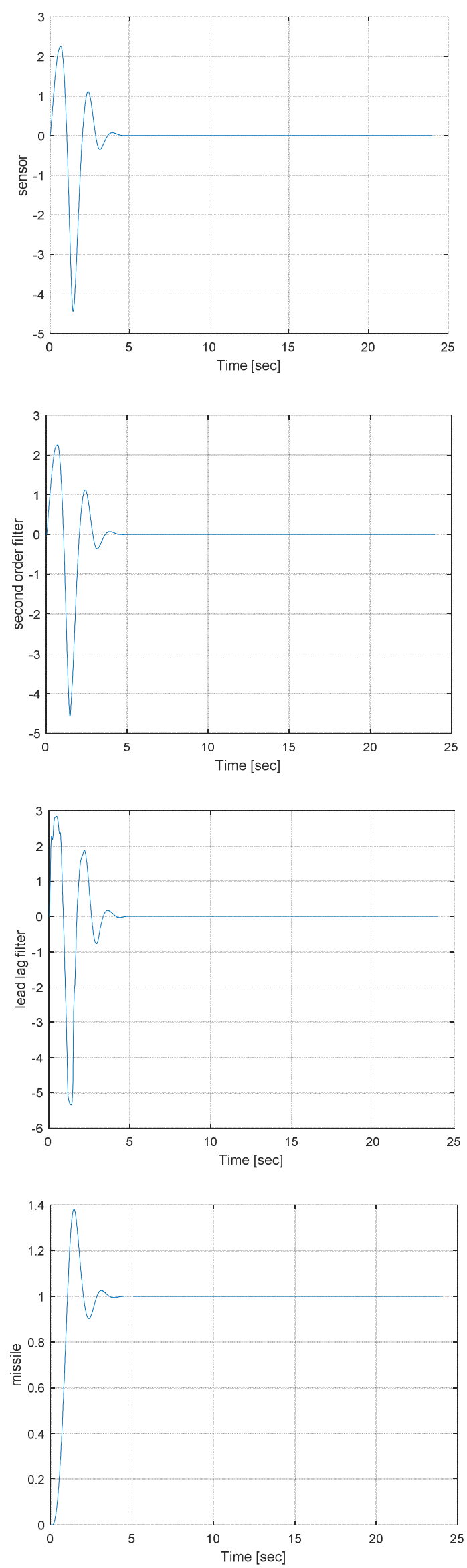

Figure 11. Yaw Plane output responses.
The simulation results show that the errors tends to zero and the missile is capable of tracking the desired input.

\section{Conclusion and Future Work}

Linearization of the mathematical representation of the underlying missile system is a milestone in investigating the behavior of the system against different flight conditions and it provides a clear picture about the system dynamics. In this paper, the transfer function of both pitch and yaw planes of the missile were derived while further investigation and analysis will be carried out in the future work to design a classical controller satisfy not only the time response analysis but also the tactical and operational specs.

\section{Abbreviations}

$\begin{array}{ll}\text { CLOS } & \text { Commanded to Line of Sight } \\ \text { AP } & \text { Autopilot } \\ \text { X } & \text { Drag force coefficient } \\ \text { Y } & \text { Lift force coefficient } \\ \text { Z } & \text { Side force coefficient } \\ 6 \text {-DOF } & \text { Six Degrees of Freedom } \\ l_{x}, l_{v}, l_{z} & \text { Characteristic linear dimensions of the missile } \\ \mathrm{S} & \text { area of wing or other characteristic area of the missile } \\ \mathrm{m}_{\mathrm{x} 1}, \mathrm{~m}_{\mathrm{y} 1}, \mathrm{~m}_{\mathrm{z} 1} & \text { dimensionless aerodynamic moment coefficient }\end{array}$

\section{References}

[1] M. A. Mohamed, "Performance Enhancemet of Autopilot Design for Short Rnage Missile using Advanced Control Techniques," Electric, Military Technical College, Cairo, 2016.

[2] N. Jack N, Missile Aerodynamics, Mountain View, USA, 1988.

[3] P. Zarcan, "Tactical and Strategic Missile Guidance," Progress in Astronautics and Aeronautic. AIAA, 1994.

[4] R. M. Goreckt, A baseline 6 degree of freedom mathematical model of generic missile, DSTO Systems Sciences Laboratory Australia 2003.

[5] G. M. Siouris, Missile Guidance and Control Systems, Ohio, USA, 2003.

[6] J. D. Anderson, FUNDAMENTALS OF AERODYNAMICS, 1991.

[7] G. El-Sheikh, Guidance System and Theory, Cairo, 2010.

[8] A. H. A. Elsayed Ahmed, A. N. Ouda, H. Eldin Hussein Ahmed, H. Mohamed Abd-Elkader, "Modeling of a Small Unmanned Aerial Vehicle," the International Journal of advanced robotic and automation vol. 4, no. 126, 2015.

[9] A. N. Ouda, "Adaptive Autopilot Design for Flying Vehicle," the International journal of dynamic and control (IJDAC), 2017.

[10] A. N. O. M. Abdallah, "Modeling and Simulation of An-Anti Tank Guided Missile" International Journal of Engineering Research and Technology vol. 7, no. 09, 2018. 
[11] L. S. a. Callaborators, Principles of Guided Missile Design, 1955.

[12] B. G. A. Ahmed Hassan Ahmed, A. N. Ouda, Ahmed Mohsen Kamel and Yehia Zakaria El-Halwagy, "Autopilot Design of Unmanned Aerial Vehicle," International journal of aeronautics and aerospace engineering, vol. 7, 2018.

[13] A. N. O. El-gabri, Ahmed S. Ahmed, Yehia Z. Elhalwagy and
Ahmed Khamis, "Command Guidance Missile Tracking Algorithms Evaluation Based On Visual Simulation," International Journal of Engineering Research and Technology, vol. 7, pp. 110-116, 2018.

[14] G. El-Sheikh and N. El. Hamwe, "Autopilot Design for a LOS Anti-tank Guided Missile," Int. Conf. Aerosp. Sci. Aviat. Technol., 2019. 